\title{
Atomic pair distribution function analysis of goethite and/or hydroxyapatite functionalized cyclodextrin nanosponges
}

\author{
Songsheng Tao ${ }^{1}$, Nangah Che Randy ${ }^{2,3}$, Ketcha Joseph Mbadcam ${ }^{2}$, Josepha Foba Tendo ${ }^{4}$, Simon J. L. Billinge ${ }^{1,5}$ \\ ${ }^{1}$ Department of Applied Physics and Applied Mathematics, Columbia University, New York, NY 10027, ${ }^{2}$ Department of Inorganic \\ Chemistry, University of Yaounde I, P.O. Box 812 Yaounde, Cameroon, ${ }^{3}$ Local Materials Promotion Authority (MIPROMALO), \\ MINRESI, P.O Box 2396 Yaounde, Cameroon, ${ }^{4}$ Department of Chemistry, University of Buea, P.O. Box 63 Buea, Cameroon, \\ ${ }^{5}$ Condensed Matter Physics and Materials Science Department, Brookhaven National Laboratory, Upton, NY 11973
}

r5nanga@yahoo.com

The local structures of four citric acid cross linked cyclodextrin nanosponges (CD); goethite (CDG), hydroxyapatite (CDHA) and goethite/hydroxyapatite (CDGHA) functionalized cyclodextrin nanosponges, prepared by ultrasound-assisted polycondensation polymerization, was studied the using atomic pair distribution function (PDF) technique. The PDFs were analyzed by comparing experimentally determined PDFs from samples under study and those from known control samples and the overall structural information extracted through visual inspections of PDFs. The samples do show the feature of cyclodextrin network linked by citric acid, with strong sharp peaks in the low-r region and weak broader peaks in the mediate and high-r region. CD and CDHA show the feature of polymer cyclodextrin, since there is only noise and density modulation after $13 \AA$ in their PDFs, while the CDG and CDGHA show the feature of crystallinity (signal even after $13 \AA$ ), which is approximately the largest atomic distance in cyclodextrin. The short-range order, which is the spacing of neighbor glucose and glucose connected by citric acid networks, is similar for all samples despite their difference in crystallization. CD and CDHA have quite similar cyclodextrin network according to their similarity in PDF while CDG and CDGHA also have cyclodextrin network but contains crystalline goethite.

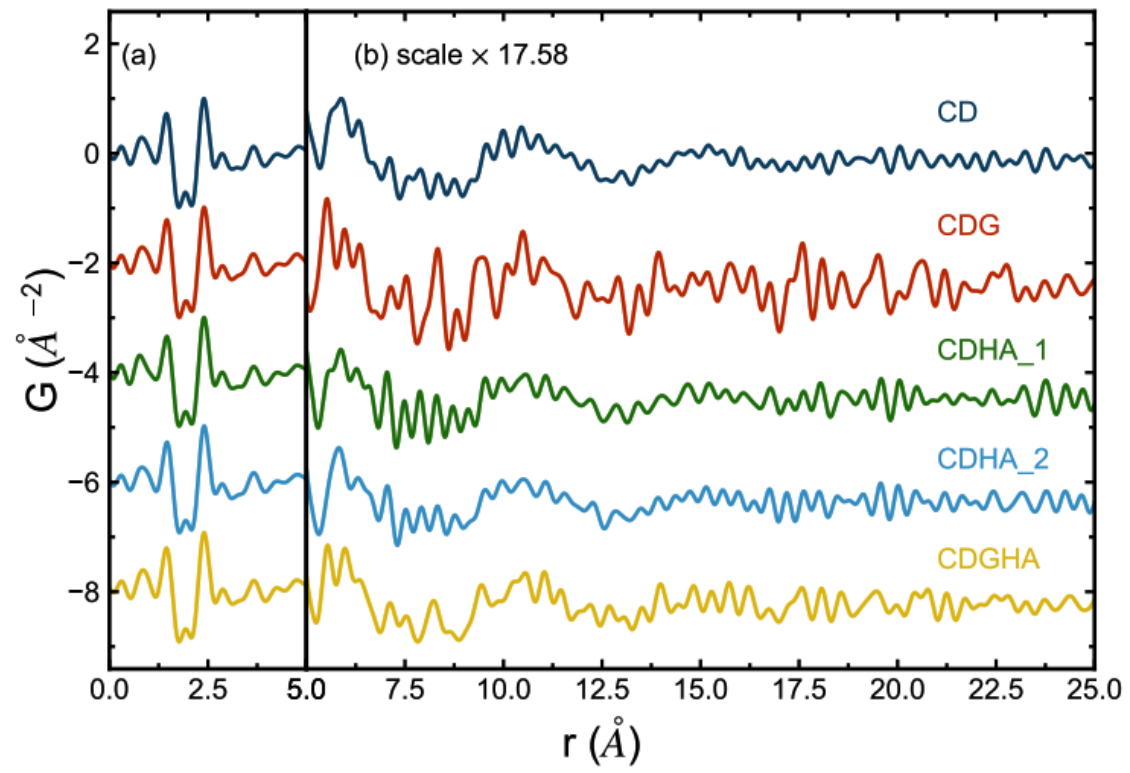

Figure 1. PDFs obtained from synchrotron x-ray powder diffraction: (a) PDFs in low-r region; (b) PDFs in high-r region, they are multiplied by the same scale for better visual inspection.

Keywords: Pair Distribution Function; nanosponge; cyclodextrin; goethite; hydroxyapatite

S. J. L. B. and S. T. were supported for PDF analysis by the NSF MRSEC program through Columbia in the Center for Precision Assembly of Superstratic and Superatomic Solids (DMR-1420634). Use of the National Synchrotron Light Source II, Brookhaven National Laboratory, was supported by the U.S. Department of Energy, Office of Science, Office of Basic Energy Sciences, under Contract No. DE-SC0012704. 